\title{
Experimental Investigation of Fastener Pull-out Response in Composite Joints under Static and Dynamic Loading Rates
}

\author{
Nikolaos Perogamvros ${ }^{1,2}$ (D) $\cdot$ George Lampeas ${ }^{3} \cdot$ Adrian Murphy $^{1,2}$
}

Received: 26 March 2019 / Accepted: 12 August 2019 /Published online: 27 August 2019

(C) The Author(s) 2019

\begin{abstract}
A novel device is adopted in order to experimentally investigate the effect of various loading rates on the pull-out response of a fastened composite joint configuration. The joint coupons comprise a composite plate made of the carbon/epoxy AS4/8552 material system and a centrally located titanium lockbolt. Tensile-type (pull) loading was applied to the specimens in a velocity range from quasi-static to $2.1 \mathrm{~m} / \mathrm{s}$. Both quasi-static and dynamic tests were conducted using the same specimen geometry and boundary conditions, which conform to international and industrial standards. The experim

ental work expands the limited literature and understanding of the mechanical response of composite pull-out joints under the action of dynamic loading. The main experimental observations revealed an increase of $15 \%$ regarding maximum load values when loading rate shifts from the static to the impact regime, while the failure patterns derived from static and dynamic tests were similar, although the latter presented a more intense damage zone.
\end{abstract}

Keywords Carbon fibre composites $\cdot$ Impact behaviour $\cdot$ Mechanical testing $\cdot$ Joints $\cdot$ Pull-out (pull-through)

\section{Introduction}

Mechanically fastened joints are widely used in the assembly of high performance and high value composite structural systems, e.g. modern aircrafts, advanced vehicular structures, etc.

Nikolaos Perogamvros

n.perogamvros@qub.ac.uk

1 School of Mechanical and Aerospace Engineering, Queen's University Belfast, Belfast BT9 5AH, UK

2 Northern Ireland Advanced Composites and Engineering Centre, Belfast BT3 9DZ, UK

3 Laboratory of Technology and Strength of Materials, Mechanical Engineering and Aeronautics Department, University of Patras, 26500 Rion Patras, Greece 
Such joints have lower structural efficiency when compared to adhesively bonded joints [1]. However, mechanically fastened joints are in general more resistant to long-term environmental degradation, and enable structural disassembly and reassembly for maintenance and repair. As mechanically fastened joints introduce discontinuities in the laminated material and the loading distribution they often are the 'weakest' elements of the structural system. Thus, fastened joints are typically critical structural elements and their design is key to structural efficiency and the load carrying capacity of the assembled structure. As a result, joint design significantly influences structural weight, structural integrity and safety throughout the service life of the structure. Beyond the initial static design of these structures, further challenges are imposed when the loading rates diverge from the quasi-static (static) regime and move towards dynamic (impact) events. Dynamic loading cases are also realistic loading-scenarios in the operating environment of advanced engineering structures (e.g. car crashes and aircraft hard landings). These loading cases are particularly important for composite joints as the failure behaviour and strength of joints can be expected to vary with loading rate, with the introduction of kinetic energy and inertia effects, along with material strain rate effects.

Although the in-plane static and dynamic behaviour of composite bolted joints has been thoroughly investigated, e.g. [2-11], only a few works exist in the experimental characterization of pure out-of-plane behaviour of composite fastened coupons, even under quasi-static tensile mode (pull loading) [12-18]. These joints, which are commonly referred as pull-through or pull-out joints, represent a very critical connection type in advanced aircraft structures and other transportation vehicles (e.g. cars or railway constructions [15]), given that the weak throughthickness properties of composite materials make them particularly vulnerable to out-of-plane failure modes. Additionally, in the new generation of aircraft fuselage designs, the thin composite skins that are usually fastened with metallic or composite stiffener parts are quite prone to pull-out failures even for cases where the loads are not directly imposed on the bolts in a tensile normal mode [16]. In a similar manner, such damage modes can also be observed during in-plane loading conditions at the final failure stages of lap joints [8,9]. However, it was only quite recently, that a relevant standard testing methodology was proposed by ASTM for such pull-through type joints in composite structures [19], while some suggestions regarding the pull-through testing geometries had been made by military standards several years earlier [20]. These methods suggest two different configurations for the pull-through testing which are commonly referred to as 'Procedure A' and 'Procedure B'. The test procedures consider a single centrally located fastener joining two flat and square specimens (Procedure A) or joining a single flat and square specimen to the loading apparatus (Procedure B). In Procedure A the specimens are offset in the in-plane orientation by $45^{\circ}$ (Fig. 1a) and the individual specimens attached to upper and lower test fixtures via specimen holes (4 in each specimen). In Procedure $\mathrm{B}$ the centrally located fastener is attached via a yoke to the loading apparatus and the specimen reacts the applied load to a rigid test fixture which is located above the specimen bottom surface (Fig. 1b). Since Procedure A is more complex, most studies use configurations based on the Procedure B; nevertheless, some variations with respect to the proposed geometrical ratios and fixture design can be observed in the literature, while some works follow industrial guidelines (e.g. Airbus, DASSAULT Aviation, etc.). The noteworthy differences between these designs are based on the boundary conditions that are applied on the laminated plate, i.e. clamped or simply supported edges, and on the various hole diameters of the jig's supporting plate (defined as 'clearance hole diameter (CHD)' in [19, 20] - see also Fig. 1b).

One of the first experimental studies was conducted by Freedman [12] who examined aluminium and composite pull-through joints with a different amount of plies; he noticed a 
(a)

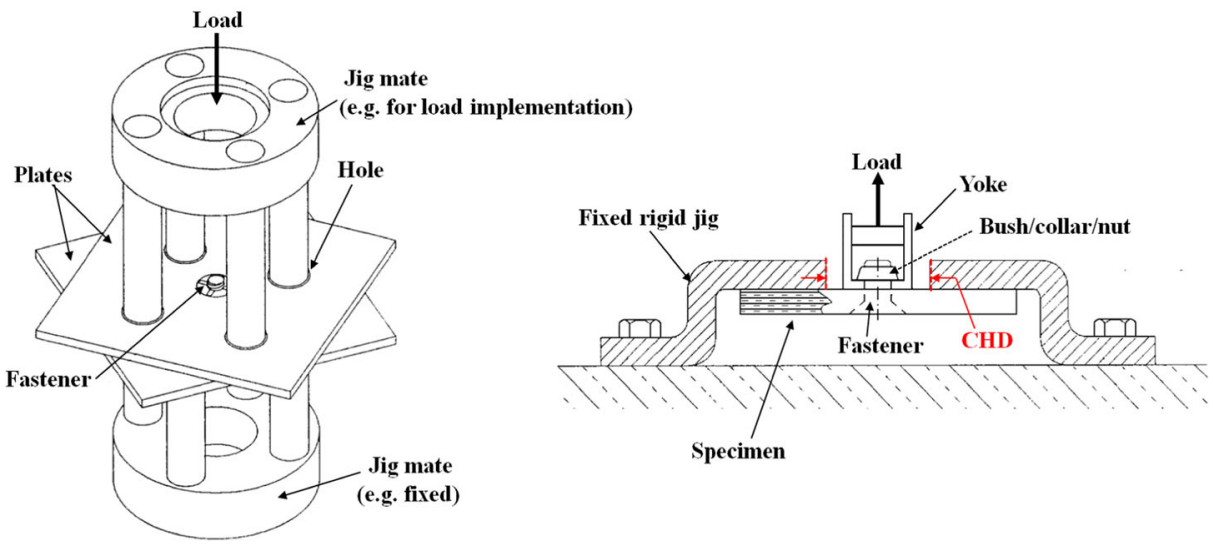

Fig. 1 Pull-through testing configurations proposed in ASTM and military standards for (a) 'Procedure A' and (b) 'Procedure B' [20]

remarkable sensitivity of the failure zone in composite samples when the ratio of the moment to through-thickness shear loading conditions changed, while aluminium plates appeared to be insensitive. Later, Banbury and Kelly [13] and Kelly and Halström [14] performed significant quasi-static test programs and failure analysis of pull-through carbon/epoxy composites. They both observed quite similar microscopic failure patterns which finally led to a pull-out damage mode. In particular, the substantial failure mode in the majority of the tested cases was the matrix shear fractures which initially occurred near the fastener head/washer area and subsequently propagated through the laminate thickness in the form of intralaminar/interlaminar shear cracks and delaminations. The damage was described as a network of cracks which extend at an inclination of $\sim 45^{\circ}$ through the thickness of the specimen laminate, akin to a three-dimensional (3D) 'staircase' failure pattern or, equivalently, a conical failure zone. Moreover, specimens with low flexural rigidity showed fibre failures at the initial damage stages in the outermost plies, especially in the bolt exit face; however, this was identified as a noncritical failure mode concerning the laminate integrity.

More recently, some additional studies [7, 8, 15-18] experimentally investigated the loaddisplacement response and the failure mechanisms of pull-out fastened configurations. A number of these works adequately commented on the concept of the initial failure load (IFL) [15-17], defined as the first load drop or a significant stiffness change in the load displacement curve. It is noted that this load value (not the maximum one) should be used in the design, as it corresponds to the onset of delamination failures which may propagate under fatigue loading conditions. Further observations on the reported load - displacement curves indicate that there are two general forms of such plots, as shown in Fig. 2 and as also stated by Pearce et al. [21]. These two forms are primarily affected by the specimen thickness and the ratio of the CHD to the fastener head diameter. Thinner specimens failed in bending when loaded with large unsupported spacing between the fastener and test fixture (resisting out-ofplane deformation), while specimens with high thickness relatively to the unsupported area failed in a shear dominated trend. Regarding the failure mode observations, similar failure patterns as the ones described in $[13,14]$ are reported. In particular, matrix cracking, delaminations and fibre breakages are the dominating failure modes. More broadly across all studies, 




Fig. 2 Typical load - displacement curves of pull-through tests published in the literature (redrawn from [21])

laminate damage is most likely at the bolt exit face with little damage observed on the fastener head side, while fastener failure is very rare.

The majority of the previous works referred to quasi-static loading conditions; with only two authors investigating the impact response of composite bolted joints during pull-through loading [7, 8]. Initially, Pearce et al. [7] tested T300/Cycom970 plain weave square plates of $65 \mathrm{~mm} \times 65 \mathrm{~mm} \times 3.52 \mathrm{~mm}$ with a centrally located $100^{\circ}$ flush head bolt and a $55 \mathrm{~mm}$ CHD support at various impact velocities, ranging from quasi-static up to $5 \mathrm{~m} / \mathrm{s}$ (some results with $10 \mathrm{~m} / \mathrm{s}$ can also be found from this research group in [21]). In their tests, limited strain rate sensitivity was observed between the quasi-static and the impact velocity testing range. The maximum forces from static tests showed elevated values compared to the dynamic results; nevertheless, slightly higher peak load values were reported as the loading rate increased from low to high rate impact tests, while the final catastrophic failure initiated slightly earlier in the higher rate tests. However, these observations should be treated cautiously, as the authors noted, since oscillation of the testing equipment is difficult to eliminate, even when moving average filters are applied to the measured data. In addition, due to the fact that different jigs and boundary conditions were used between the quasi-static (clamped) and the dynamic (simply supported) tests, the results cannot be easily compared in terms of load - displacement curves. With respect to failure mode observations, similar failure patterns were found between statically and dynamically failed samples; in particular, delamination and matrix shear failures, as well as ply splitting on the bolt exit face were mainly observed.

In the other dynamic study, Heimbs et al. [8] experimentally investigated HTS/Cycom9772 composite rectangular plates ( $2 \mathrm{~mm}$ thickness) with $130^{\circ}$ flush head titanium bolts at three different velocities, i.e. quasi-static, $2 \mathrm{~m} / \mathrm{s}$ and $10 \mathrm{~m} / \mathrm{s}$. In these tests, the specimens were clamped in a way leading to a free square bending area of $28.8 \mathrm{~mm} \times 28.8 \mathrm{~mm}$. Identical boundary conditions were applied in both static and dynamic tests using the same grip which was installed in a Zwick high speed servo-hydraulic machine. The results indicated no distinct strain rate effects of the pull-through specimens. Specifically, the authors of [8] noted that the global shape of the dynamic and quasi-static data in terms of initial elastic behaviour, damage 
initiation and the post damage drops in load were all comparable. However, the unfiltered dynamic curves suffered from some oscillations which were mainly attributed to the piezoelectric load cell position and its clamp attachments; this effect produced fluctuated load signals, with low and high peak values which notably diverge from the static curve. Concerning failure observations, delaminations and matrix cracks that were followed by fibre breakages were the main damage patterns in both static and dynamic tests.

Nevertheless, the lack of experimental data regarding the pull-out configurations which adopt standardized identical geometry and boundary conditions between static and dynamic tests, the problems of the untreated signal oscillations of the existing dynamic data, the contradicting results of the loading rate effects on the joint's response, and the necessity of investigating other composite material systems, indicate the need of further examination of these important joint types. With the aim to bridge the aforementioned gaps and provide a further insight into the actual mechanical response of composite pull-out joints, the work herein presents the test of a typical aerospace material system and fastener combination (carbon/epoxy AS4/8552 and titanium countersunk lockbolts) subjected to a velocity range, i.e. from quasi-static to $2.1 \mathrm{~m} / \mathrm{s}$, representative of several aircraft loading conditions, such as hard landing and ditching events [22, 23]. To achieve the dynamic tests, a novel test fixture [24] and a standard drop tower test machine is used. The work therefore also demonstrates a setup and test procedure to perform joint pull tests without a dedicated high speed servo-hydraulic test machine.

\section{Experimental Configurations}

\subsection{Material System and Specimen Configuration}

In the present work testing is based on the 'Procedure B' (ASTM and military standards $[19,20])$, as described in the Introduction section, and pull-out (PO) type composite specimens. The specimens are manufactured from unidirectional carbon/ epoxy material (AS4/8552) with each specimen having a quasi-isotropic stacking sequence of $[45 / 90 /-45 / 0]_{2 \mathrm{~s}}$. The final cured and trimmed dimensions of the individual specimens are $50 \mathrm{~mm} \times 50 \mathrm{~mm} \times 2.4 \mathrm{~mm}$. A centrally located countersunk lockbolt fastener, made of aerospace grade titanium alloy Ti-6Al-4V (pin material) and aluminium alloy 2024-T42 (bush material), connects the laminate with a hollow cylindrical part made of steel (Fig. 3). The latter metallic component has a threaded lower portion and is used for the load implementation in a similar manner as the rigid yoke proposed in the standardized procedures. The specimen design and its dimensions are shown in Fig. 3 and Table 1. It should be noted at this point that the selected coupon design is a representative fastened area of a fullscale hybrid composite-metallic fuselage section of a Dassault Falcon business jet. This connection type, which may also be found in several new aircraft types, is particularly prone to the pull-out failure mode since the thin composite skin presents weak through-thickness properties compared to its metallic mates (stringers/stiffeners). Thus, the adopted specimen design in conjunction with the developed supporting/loading jig (see section 2.2 for further details) aims to provoke such types of final macroscopic failure modes. As part of SMAES (SMart Aircraft in Emergency Situations) research project, the assembled specimens were designed and provided by DASSAULT Aviation [25]. 


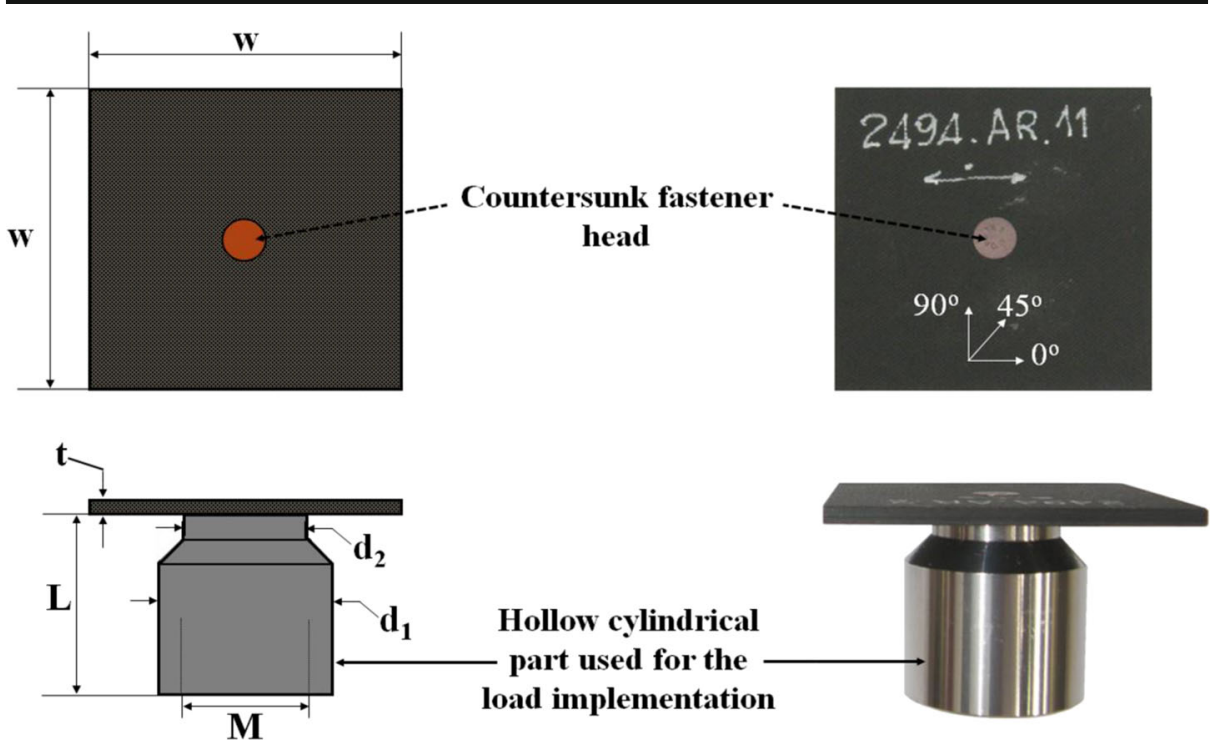

Fig. 3 PO specimen design, dimensions and laminate coordinate system

\subsection{Testing Apparatuses and Equipment}

Two load frames were used for specimen testing, an MTS $100 \mathrm{kN}$ servo-hydraulic testing machine for the quasi-static tests, and a specially designed test arrangement within an Instron $9250 \mathrm{HV}$ drop tower testing machine for the dynamic (impact) tests. The crosshead of the Instron machine is capable of achieving a velocity of up to $20 \mathrm{~m} / \mathrm{s}$ and a maximum energy level of up to $1000 \mathrm{~J}$.

Drop tower test machines are designed primarily for dropping a specific mass on a structural specimen or component in order to derive understanding of its crushing and collapse behaviour. In order to perform the desired tensile-type pull tests using such a drop tower machine a new device was designed and implemented during testing (described hereafter as the 'Tension_device'). Using such a unique fixture with a standard drop tower machine for dynamic loading provides a very cost effective alternative to purchasing and operating a very specialised and expensive high speed servo-hydraulic testing machine. The special fixture, originally developed in a previous work [24], and successfully employed for dynamic lap-joint tests [26], is illustrated in Fig. 4. The standard design of the Tension_device consists of two steel frames, one of which is motionless (labelled rigid as it does not move) and a second movable frame. The Tension_device concept is independent of the drop tower machine but the specific geometry of the frames is defined around the Instron machine noted above (its geometric envelope and the loading range). In addition, there are a series of steel grips attached to each frame. The upper grips are connected to the upper plate of the motionless frame, while the lower grips are connected to the bottom plate of the movable frame. When the drop tower

Table 1 PO specimen nominal dimensions

\begin{tabular}{llllllll}
$\mathrm{W}(\mathrm{mm})$ & $\mathrm{t}(\mathrm{mm})$ & $\mathrm{L}(\mathrm{mm})$ & $\mathrm{M}(\mathrm{mm})$ & $\mathrm{d}_{1}(\varnothing)(\mathrm{mm})$ & $\mathrm{d}_{2}(\varnothing)(\mathrm{mm})$ & $\mathrm{d}_{\text {fastener }}(\varnothing)(\mathrm{mm})$ & Countersunk angle $\left(^{\circ}\right)$ \\
\hline 50 & 2.4 & 30 & $20 \times 1.5$ & 28 & 19.5 & 4 & 100 \\
\hline
\end{tabular}


impactor strikes the upper plate of the movable frame, the specimen is the load path between the two frames. The combination of frames enables the transformation of the compression loading mechanism into a tension loading mechanism between the upper and lower grips. In order to minimise the friction effects and ensure alignment between the two frames, bronze rings are used in the contact areas between the two frame systems. Additional alignment features (cylindrical rods) are also located between the bottom plate of the movable frame and the base plate of the rigid frame. Together these features ensure that the movement of the movable frame is parallel to the rigid frame and the specimen between the grips is loaded appropriately.

In order to measure specimen loading a piezoelectric load cell ( $\pm 60 \mathrm{kN}$ KISTLER 9361B) is positioned in the load path between the motionless frame and the specimen grips. To measure displacement a non-contact linear variable differential transformer (LVDT) is also integrated into the design and is placed to measure axial deflection between the moveable frame bottom plate and the rigid frame (Fig. 4). Load and deflection measurements are captured using a data acquisition system (DAQ) from National Instruments with a maximum scan rate of $5 \mathrm{MHz}$. The Labview software is used to manage initial data recording. Finally, in order to protect the drop tower machine (in particular the load cell), an energy absorbing material is positioned on the top of the movable frame.

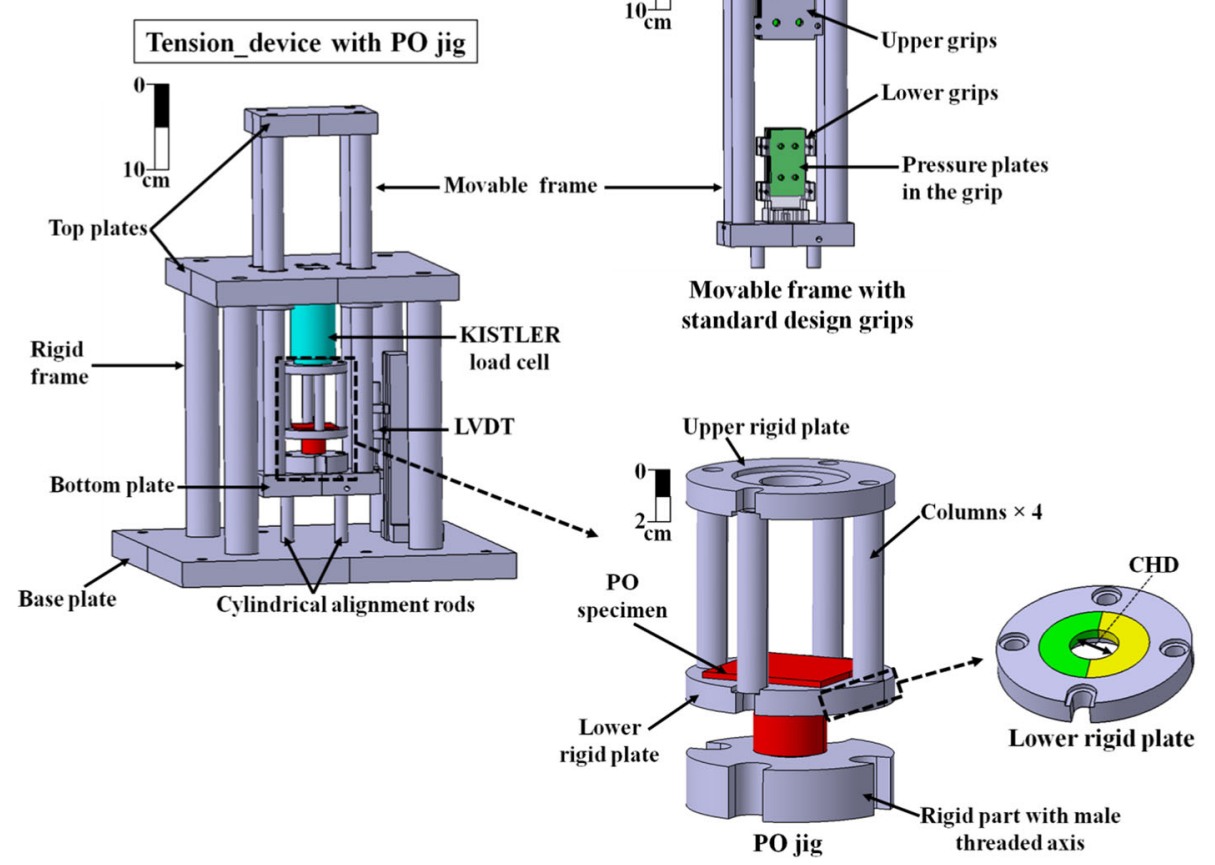

Fig. 4 Tension_device in conjunction with PO jig - Tension_device's movable frame with standard design grip assemblies (CAD representation using CATIA V5) 
However, in the current work, in order to accommodate the PO specimens and apply specific loading and boundary conditions on this coupon configuration, the standard Tension_device grips were replaced with a steel jig (referred also as PO jig) which was designed and manufactured as shown in the lower portion of Fig. 4. The main components of the PO jig are the two circular rigid plates (upper and lower), which are joined together with four bolted columns, and a separate rigid part which contains a male threaded axis for the connection with the hollow cylindrical part of the specimen. This jig can be used in a standard test machine (herein in the MTS machine) or in the Tension_device such that a series of dynamic and static tests can be performed with a single local test setup on different test machines - eliminating possible discrepancies due to local fixture differences. In the current design the composite laminate specimen is simply supported on the lower plate of the PO jig, with an unsupported area (CHD) of $20 \mathrm{~mm}$ (Fig. 4). With the PO test arrangement, the tensile load of the test machine or Tension_device loading plates results in a tensile-type (pull) load on the fastener. The fastener transfers the load to the specimen, which results in an out-of-plane reaction loading between the composite part of the coupon and the lower rigid plate of the PO jig (Fig. 4). This, out-of-plane, loading condition on the square plate creates significant compressive stresses in the material under the fastener's head and elevated bending moments in the unsupported circular area of the laminate. Geometric constraints on the PO design due to the requirement to fit two test arrangements means that the usable specimen and jig dimensions vary slightly from that proposed in the ASTM standards for static tests. However, the minimum laminate thickness and the boundary principles are maintained to be very similar. Moreover, the above PO test set-up conforms to the standard defined by the partner airframer and test specimen provider (DASSAULT Aviation).

In order to capture the specimen behaviour during test, the digital image correlation (DIC) technique is used. For the static tests an ARAMIS 5M system is employed (along with the GOM software), while for impact tests a Dantec Dynamics setup is adopted. During the dynamic tests the cameras were recording at $20 \mathrm{k}$ frames per second (fps). The non-optical measuring devices, i.e. LVDT and KISTLER load cell, were set to acquire data at $80 \mathrm{kHz}$. In the case of the quasi-static test the DIC cameras were set to acquire images with $1 \mathrm{fps}$.

Pre and post-test non-destructive evaluations were completed for a number of specimens using the C-scan technique (with the aid of ULTRAPAC system and ULTRAWIN software). In order to achieve high quality measurements, a $10 \mathrm{MHz}$ flat transducer with a diameter of 0.25 -in. was applied and the inspection undertaken with the coupons immersed in distilled water. Optical microscopy was also undertaken on selected specimens in their post-test state (using a microscope Leica DM LM). The combined ultrasonic and optical inspections were used to examine failure surfaces and identify modes of failure.

\section{Experimental Procedure, Results and Discussion}

Several PO specimens were tested at various loading rates (i.e. five samples per rate). In particular, two dynamic loading rates were considered along with a base quasi-static test. Repeatable results were found for each loading rate. For the dynamic tests the Tension_device was located in the drop tower test machine and the PO jig fixed into the Tension_device; afterwards, the coupons were placed into the PO jig (Fig. 5a). To achieve the two dynamic loading rates, two energy levels ( $100 \mathrm{~J}$ and $400 \mathrm{~J}$ ) were applied to the experimental setup using the drop tower machine. This resulted in two crosshead velocities, 3.3 and $6.5 \mathrm{~m} / \mathrm{s}$. However, 
the loading rate transmitted through the setup to the specimen is reduced due to the energy absorbing material positioned on the top of the movable frame. Thus, specimen loading rate (actual velocity) is measured and presented using only the LVDT and the DIC data.

The base quasi-static test was performed with the PO jig located in the servo-hydraulic testing machine with special adapter fittings; then the specimens were individually placed into the PO jig before each test (Fig. 5b). Displacement loading was applied at $1 \mathrm{~mm} / \mathrm{min}$. Load and cross-head displacement data was recorded using the integrated test machine measurement system at a sampling rate of $100 \mathrm{~Hz}$.

Figure 6a presents typical quasi-static and dynamic raw test results in the form of load versus displacement plots. The first observation in this plot is the high amplitude oscillations seen in the dynamic test curves which may significantly mask the true loading history. The spurious effect on the 'true' response of the specimen is possibly caused by the rigid PO jig which is fixed on the piezoelectric load cell of the Tension_device (Figs. 4 and 5a). This arrangement changes the load train components and the attached masses of the original gripping design (standard grips in Fig. 4), suggesting that further analysis and design to the setup could improve the experiment. For example, significant eigenvalue analyses were conducted during the development of the Tension_device with the standard grip assemblies showing that such effects can be minimised through careful analysis and design $[24,26]$. However, the specific PO specimen and jig configuration are currently developed for compatibility reasons to the industrial standards. It is worth noting that this is not a unique challenge as noisy results are observed in the dynamic data of the most relevant works that are available in the literature $[7,8]$. Aiming to remove a part of these oscillations and achieve a more reasonable comparison against the quasi-static data, a higher-order polynomial regression filter which utilizes a quintic (5th order) function was applied on the raw force data; the use of such higher degree polynomial filtering enables an appropriate level of data smoothing but without causing attenuation of the data features. An analogous filtering technique was also applied by the authors in [7]. Polynomial filtering techniques are well established and documented, available in commercial software such as Matlab, and the interested reader is directed for further details to the relevant literature, e.g. [27]. The resulting 'smoothed' load displacement plots are depicted in Fig. $6 \mathrm{~b}$ and will be used in the forthcoming discussion on
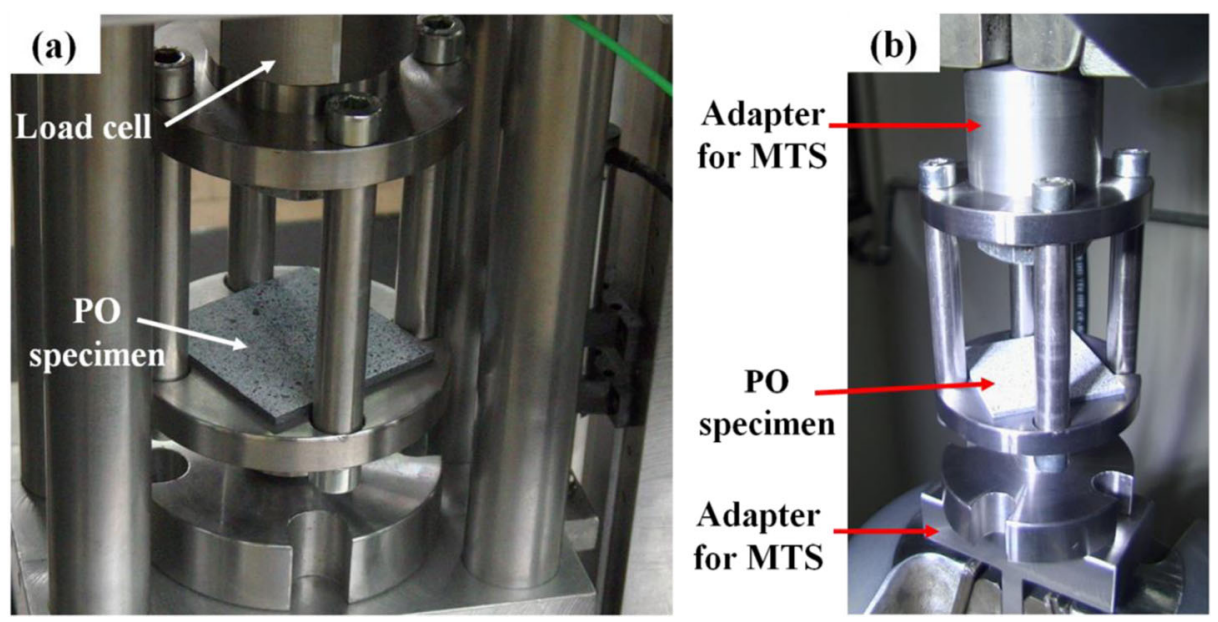

Fig. 5 PO jig with the relative specimen (a) placed in the Tension_device and (b) fixed in the MTS grips 
the response of the PO specimens. However, the introduction of data smoothing will again be discussed when general trends are drawn as specimen dynamic responses have this identified uncertainty.

In Fig. $6 \mathrm{~b}$ it can be clearly observed that both the static and the dynamic curves initially show an almost linear elastic region, which is related to the bending phase of the composite specimen. Afterwards, in the static tests, a sudden load drop is illustrated, indicating that the IFL is reached. This abrupt drop of the force is closely related to rapid shear fractures in the matrix, which initially occurs under the fastener head area in a form of interlaminar (delamination) or intralaminar damages, as also reported in the relevant literature [13, 14]. This behaviour during static tests, which correlates well with the corresponding trend in Fig. 2, is expected since the ratio of CHD to the fastener head diameter is below 3, thus provoking a shear dominated response [15]. Subsequently, the statically obtained load values increase again, following a second slope that shows a lower stiffness compared to the initial elastic stiffness. This stiffness is maintained until the maximum load value is reached. After this point, the force is linearly scaled down towards zero values, where the bolt is totally removed from the composite plate. Concerning the load - displacement plots of the dynamically tested specimens, some discrepancies may be observed after the initial elastic response compared to the statically tested samples, as shown in Fig. 6b. In particular, the dynamic curves do not present a discrete IFL value, while the ultimate load is achieved after the initial elastic stiffness zone. This difference may be caused by the nature of the impact tests, in which the initiation and the accumulation of damage take place almost simultaneously. In addition, after this point of maximum force data, the load values are linearly scaled down until a relatively high value $(1.5-3 \mathrm{kN})$ and then a sudden load drop follows, indicating that the joint has no further capability to carry loads. The latter effect leads to smaller ultimate displacement values in the dynamic tests compared to the static ones. Similar observations were also reported in [8], while Pearce et al. in [7] have published some contradicting results; nevertheless, it is important to note that in the latter publication [7] different boundary conditions were used between static and dynamic tests. Further details on the displacement discrepancies are provided in a following paragraph of this section, where the relevant failure modes are discussed.

With respect to the maximum load values, no rate sensitivity is observed in the impact results, while a small level of sensitivity is identifiable between the static and dynamic cases. To better quantify the static to dynamic sensitivity, Fig. 7 presents the maximum load (mean value with standard deviation error bar) versus the actual velocity data. From the latter figure it
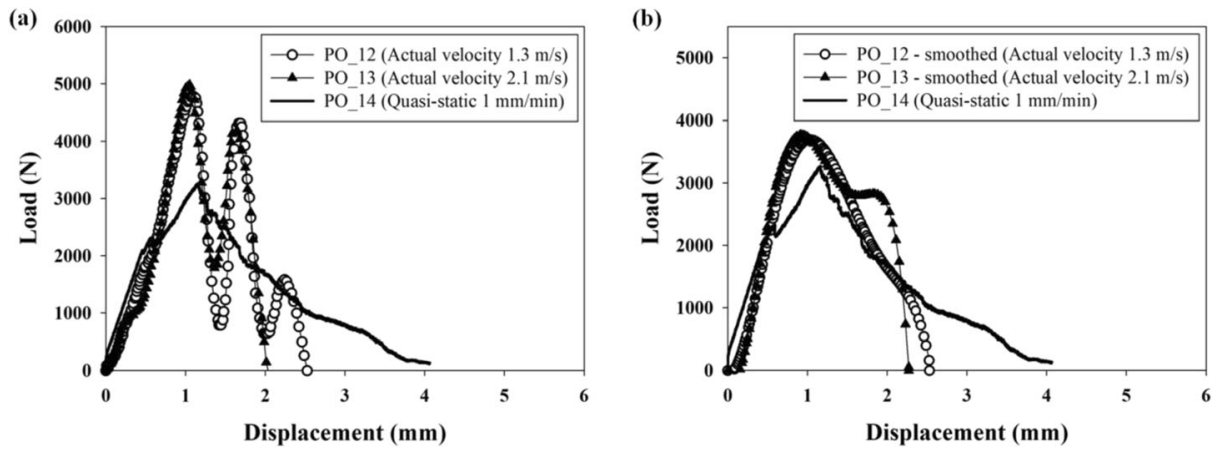

Fig. 6 Representative load - displacement curves of the PO tests; curves extracted from dynamic experiments are based on (a) raw and (b) filtered data 
can be seen a measured variation of the order of $15 \%$ between the quasi-static and the dynamic results, and only a negligible variation of approximately $2 \%$ between the individual dynamic test results. These variations may be attributed to strain rate effects of the resin material when subjected to rapid deformations; further details are also provided in a later paragraph of this section, after the failure modes assessment, which is discussed next.

To better understand the similarities and discrepancies between the static and the dynamic cases, it is necessary to examine in detail specimen responses during test, as well as the observation of the specimen failure modes. Figure 8 presents camera footage during test for the static and one of the impact tests $(1.3 \mathrm{~m} / \mathrm{s})$. Figure 9 presents post-test images of the same test combination. Examining Fig. 8, both the statically and the dynamically tested coupons display a similar initial bending phase which causes the elevation of the simply supported laminate edges (no contact with the rigid supporting plate); however, in the static test this effect is also observable after the IFL point (Fig. 8a). At this point, the composite plate edges are marginally lowered, but some level of clearance still exists. Additionally, some local bending effects are developed in the CHD area of the specimen (Fig. 8a). These effects appear in all statically tested coupons during the second slope part of the loading phase, until the maximum load is reached. After this phase, the fastener penetrates the laminate and the composite plate then returns towards its initial position, while the joint response moves into the unloading phase. Contrastingly, this discrete transition between the two bending slopes is not observable in the dynamic tests, as also previously mentioned. This behaviour can be explained with the aid of Fig. $8 \mathrm{~b}$, where it can be clearly seen that after the dynamic bending phase, the composite plate immediately returns to the initial position and, almost simultaneously, the fastener head penetrates the laminate, defining the start of the unloading curve.

Nonetheless, the rather similar load - displacement general trends and the small loading rate sensitivity between static and dynamic experiments may be attributed to the comparable failure patterns observed in all tested samples, as shown in Fig. 9. More specifically, all failed PO specimens (dynamically or statically tested) exhibit a splitting pattern on the exit face of the fastener (bottom view - Fig. 9) with two diagonal cracks in the $45^{\circ}$ orientation (near the fastener hole), along with two raised petals. The composite raised sections are probably a result of extensive matrix failure (e.g. delamination and shear cracks), as well as fibre splitting at the lower portions of the laminate, over an area which is much larger than the fastener's head

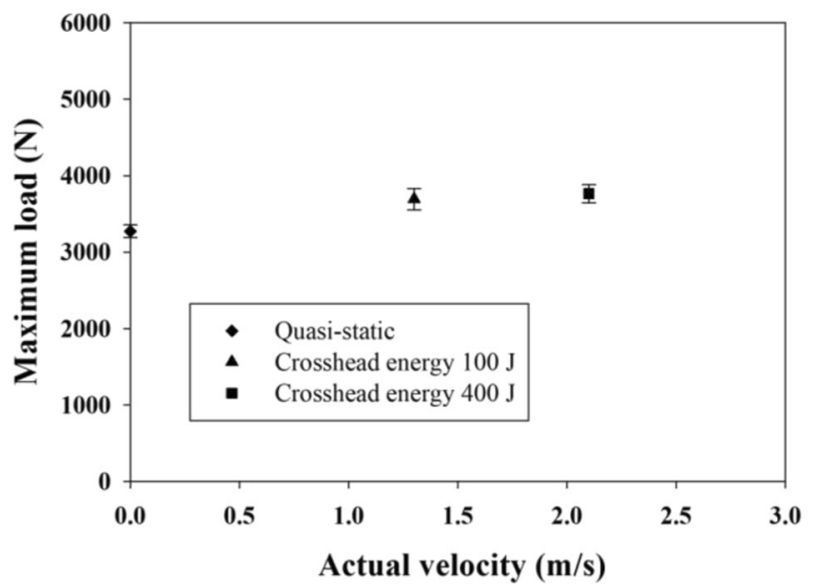

Fig. 7 Maximum load (mean value with standard deviation error bar) versus actual velocity data of the PO tests 
(a)
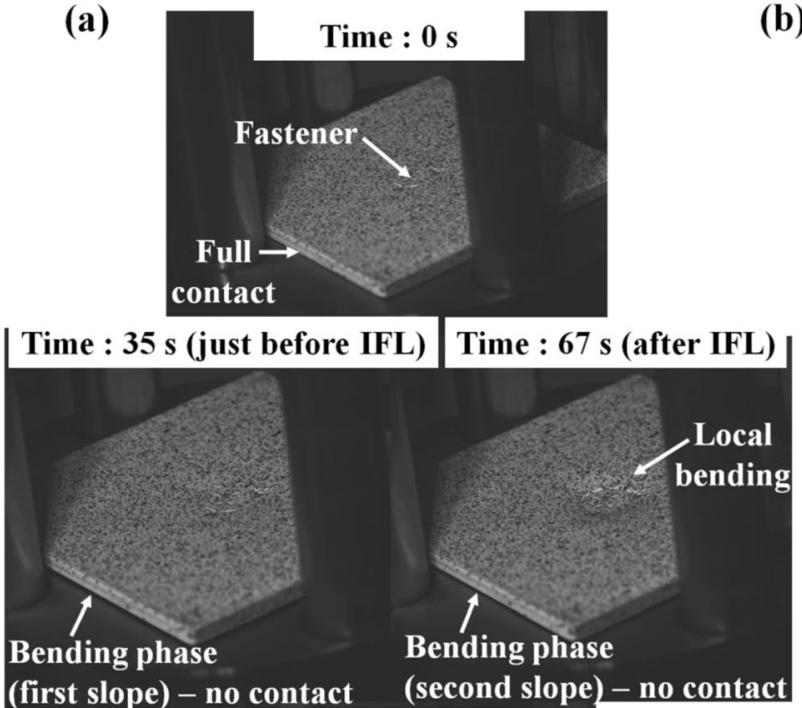

(first slope) - no contact (second slope)- no contact

Time : $72 \mathrm{~s}$

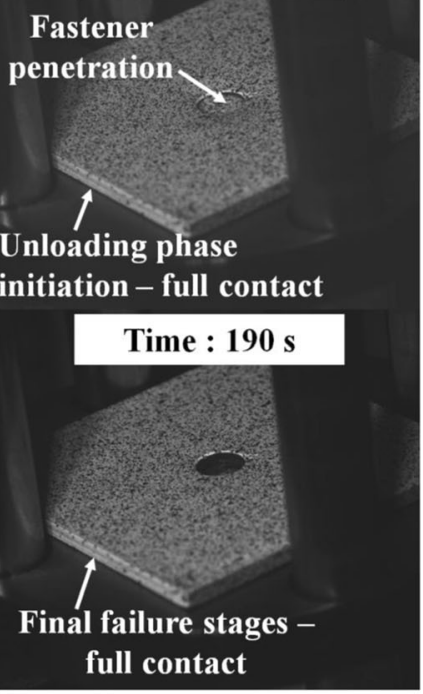

(b)

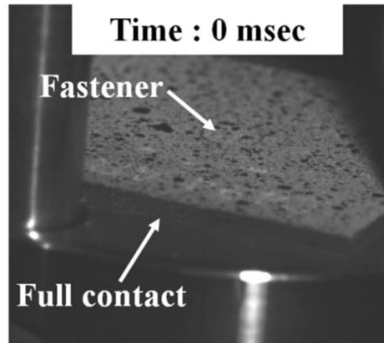

Time : 0.4 msec

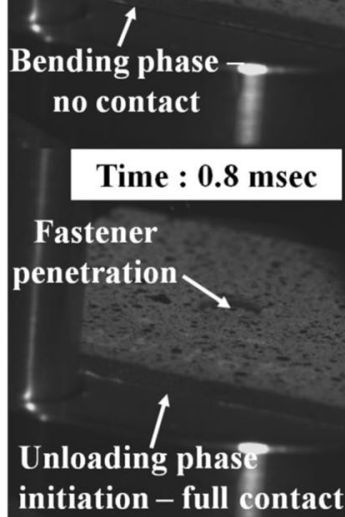

Time : $1.7 \mathrm{msec}$

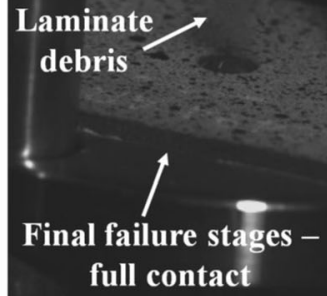

Fig. 8 (a) Low and (b) high speed camera footages of a static and a dynamic $(1.3 \mathrm{~m} / \mathrm{s})$ PO test, respectively

maximum cross-section. Moreover, in all these cases, the laminate at the entry face of the fastener (top view - Fig. 9) appears to be undamaged, with a neat hole of the size of the pin head. This is similar to the post-test failure modes reported in the relevant literature [7, 16]. Another common observation between static and dynamic PO tests is the undamaged fasteners (Fig. 9).

Figure 10 presents the C-scan contours of both the statically and dynamically tested samples. Examining Fig. 10 it is observable that the composite material is extensively damaged (e.g. delaminations, matrix cracks, etc.) in a circular area, the diameter of which is approximately equal to the CHD value. The latter observation confirms the above mentioned remarks concerning the failure mechanisms that are 


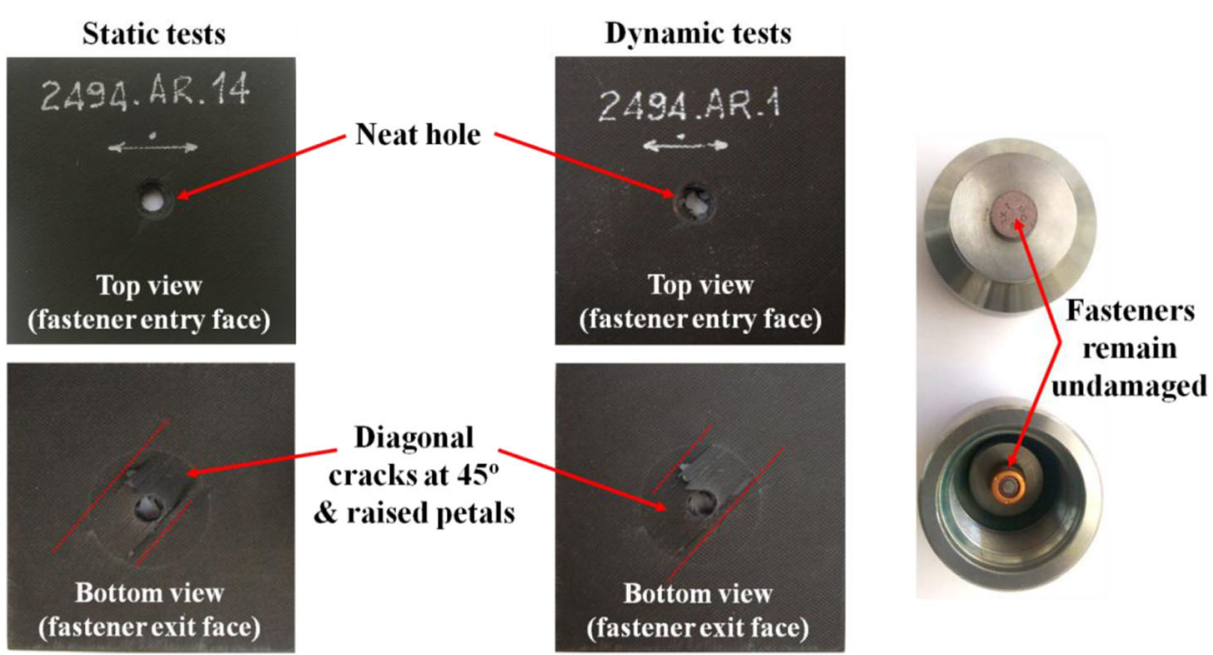

Fig. 9 PO specimens' failure modes at the post-test state - laminate and fastener top/bottom views

involved in the creation of the composite raised petals. In addition, the circular failure pattern is more intense in the case of the dynamically loaded samples.

Moreover, aiming to identify the developed failure modes in the through-thickness direction of the laminate, micrographs of arbitrary cross-sections which run through the hole boundary area are examined and shown in Fig. 11. It can be observed in these plots that both the statically and the dynamically tested samples present similar failure mode trends. In particular, a 'staircase' damage pattern is observed comprising transverse shear matrix cracks and fibre breakages which usually lead to delamination failures; this failure trend is also reported in the relevant literature (e.g. $[13,16])$. In addition, the lower plies, or a group of them, present extensive fibre splitting under pronounced bending loads, which are mainly caused by the pin head, indicating in this way that the final failure stages have been reached. However, some differences are observed between the dynamic and static tests with respect to the latter failure pattern. Specifically, in the static coupon, it seems that the plies are initially separated and then some single plies undergo fibre splitting, while in impacted samples a thicker sublaminate part (not separated plies) fails under the respective final load. This effect, which can be caused by the stiffening tendency of the compressive through-thickness composite material properties at elevated strain rates, provides an

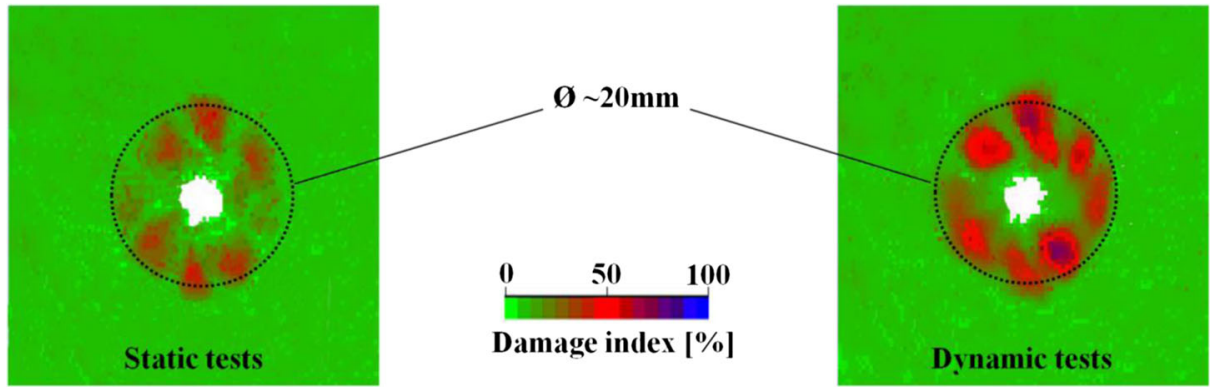

Fig. 10 Indicative C-scan contours of PO samples tested under static and dynamic loading conditions 
explanation for the noticed discrepancies in the displacement readings (Fig. 6b) as the loading rate moves from quasi-static to dynamic. It is important to mention that the compressive through-thickness composite response is generally affected by the matrix material properties; these properties commonly present strain rate dependency regarding their stiffness and strength values $[28,29]$. This strain rate dependency might also be responsible for the somewhat elevated maximum force levels between static and impact tests (Fig. 7). Nevertheless, this is not a general outcome for composite joints and laminated systems. The rapid deformation of the matrix-rich areas in these complicated structural elements can also lead to elevated material temperatures [28]; such temperature effects can act in a contradicting manner to the stiffening tendency of the matrix constituent, resulting in opposite load trends to those described above [26, 30]. In [26] strength values of filled-hole coupons and single-lap joints are documented, while in [30] data regarding interlaminar strength properties can be found; both investigations utilize the same laminate system used in the current pull-out study.

With the aim to evaluate the above observations from the PO tests in terms of crash resistance, the energy absorption, extracted by integrating the area under the load - displacement curves (Fig. 6b), is presented in Fig. 12 (mean value with standard deviation error bar). Examining Fig. 12, again there appears to be limited sensitivity between the static and the dynamic results. This is expected given the earlier findings with respect to the load - displacement behaviour and the underlying similar physical failure behaviours. Nevertheless, the somewhat lower energy absorption in impacted specimens compared to static data $(\sim 7 \%)$ is attributed to the smaller ultimate dynamic displacements, which counteract the elevated maximum load values observed in dynamic tests. It is also necessary at this point to consider the smoothing applied to the dynamic results, which may slightly alter the actual coupon's response indicating the need of further refinements in the standardized pull-through arrangements in order to be adapted for impact testing.

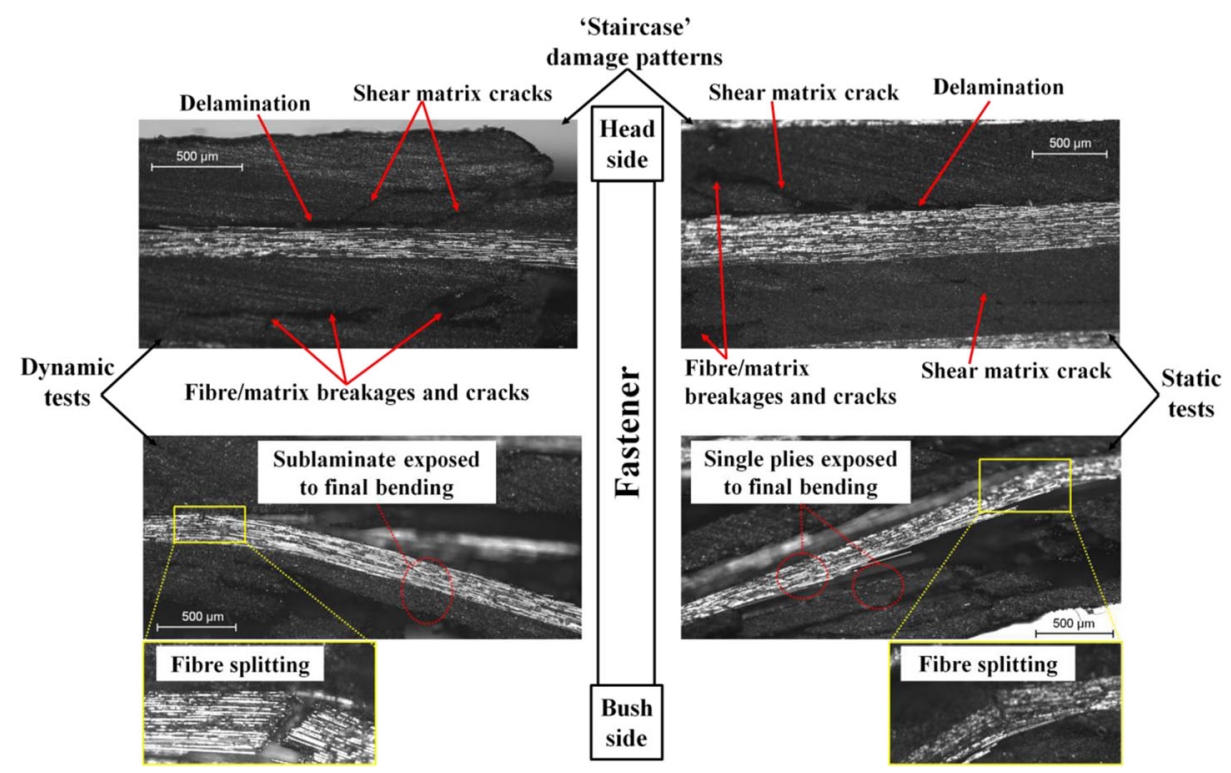

Fig. 11 Identification of the through-thickness microscopic failure modes in static and dynamic PO tests 


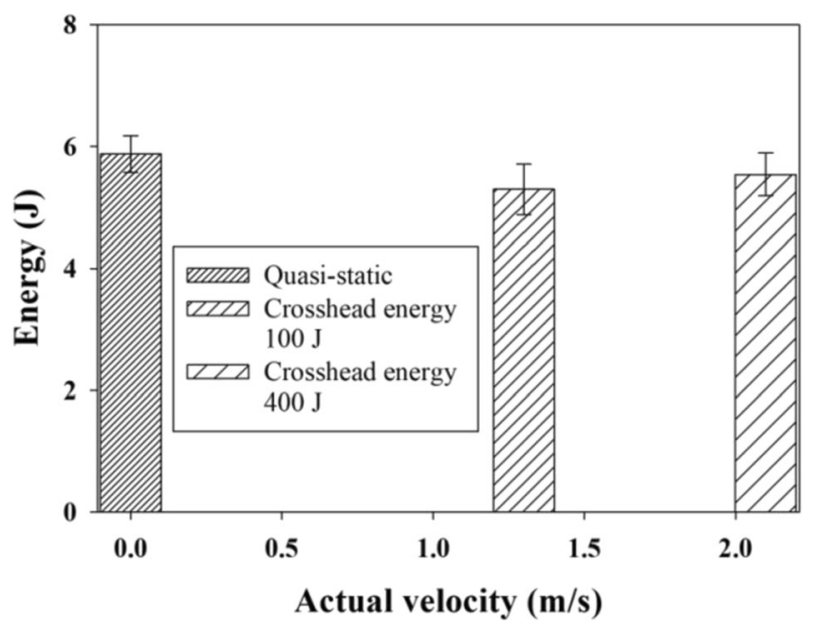

Fig. 12 Energy absorption (mean value with standard deviation error bar) of the PO specimens at each loading rate

\section{Conclusions}

In this work the behaviour of a composite fastened joint configuration (PO joint), designed to provoke pull-through damage, was investigated through physical testing in order to assess the effects of the loading rate. The tests were performed under various velocities, ranging from quasi-static, i.e. $1 \mathrm{~mm} / \mathrm{min}$, to $2.1 \mathrm{~m} / \mathrm{s}$ impacts with the aid of an innovative testing device which enables the use of a drop tower for tensile-type loading. Additionally, a jig (PO jig) was developed and incorporated into the tensile testing device to provide appropriate specimen gripping and meet the requirements set by the industrial test standard defined by the material provider. The load - displacement response, the energy absorption capability and the failure mode of the joints where systematically examined. The test results demonstrate a small level of load rate sensitivity, i.e. an increase of about $15 \%$, in terms of strength values between the statically and dynamically tested coupons. Larger discrepancies were identified with respect to the ultimate displacement readings which follow a decreasing trend. As a result, the specimen energy absorption illustrated limited sensitivity, i.e. up to $7 \%$, for the different loading rates, since the elevated maximum load values counteract the smaller ultimate displacements in the case of the dynamic test results. Regarding the specimen failure modes, all PO specimens (dynamically or statically tested) exhibited similar failure patterns with extensive matrix failures, such as delaminations and shear cracks, as well as fibre splitting in the lower side of the plate laminate. However, the aforementioned failure patterns were more intense having slightly different sublaminates/plies' failure behaviour in the case of the impacted samples, providing a possible explanation of the differences in load - displacement response. Moreover, it is important to mention that further developments should be considered on the design of the gripping jig/specimens of the standardized pull-through configurations due to the possible introduction of spurious 'noise' on the actual specimen dynamic response. The presented testing methodology can be extended for the derivation of the impact response at higher impact velocities and strain rates, as well as to other composite material systems, taking into account that pull-through joint failures are commonly met in typical crash events of advanced composite structures and are closely related to the weak through-thickness properties of composite materials. 
Acknowledgements The current research work is partially supported by the EU, in the frame of 'SMart Aircraft in Emergency Situations' (SMAES) - RTD project, Contract No. ACP0-GA-2010-266172. The authors would also like to gratefully acknowledge DASSAULT Aviation and Dantec Dynamics for providing the joint specimens and the 3D high speed optical system, respectively.

Open Access This article is distributed under the terms of the Creative Commons Attribution 4.0 International License (http://creativecommons.org/licenses/by/4.0/), which permits unrestricted use, distribution, and reproduction in any medium, provided you give appropriate credit to the original author(s) and the source, provide a link to the Creative Commons license, and indicate if changes were made.

\section{References}

1. Sharos, P.A., Egan, B., McCarthy, C.T.: An analytical model for strength prediction in multi-bolt composite joints at various loading rates. Compos. Struct. 116, 300-310 (2014)

2. Starikov, R., Schön, J.: Quasi-static behaviour of composite joints with countersunk composite and metal fasteners. Compos. Part B Eng. 32, 401-411 (2001)

3. McCarthy, M.A., Lawlor, V.P., Stanley, W.F.: An experimental study of bolt-hole clearance effects in singlelap, multibolt composite joints. J. Compos. Mater. 39, 799-825 (2005)

4. Ataș, A., Soutis, C.: Subcritical damage mechanisms of bolted joints in CFRP composite laminates. Compos. Part B Eng. 54, 20-27 (2013)

5. Yazdani Nezhad, H., Egan, B., Merwick, F., McCarthy, C.T.: Bearing damage characteristics of fibrereinforced countersunk composite bolted joints subjected to quasi-static shear loading. Compos. Struct. 166, 184-192 (2017)

6. Portemont, G., Berthe, J., Deudon, A., Irisarri, F.X.: Static and dynamic bearing failure of carbon/epoxy composite joints. Compos. Struct. 204, 131-141 (2018)

7. Pearce, G.M., Johnson, A.F., Thomson, R.S., Kelly, D.W.: Experimental investigation of dynamically loaded bolted joints in carbon fibre composite structures. Appl. Compos. Mater. 17, 271-291 (2010)

8. Heimbs, S., Schmeer, S., Blaurock, J., Steeger, S.: Static and dynamic failure behaviour of bolted joints in carbon fibre composites. Compos. Part A. 47, 91-101 (2013)

9. Egan, B., McCarthy, C.T., McCarthy, M.A., Gray, P.J., O’Higgins, R.M.: Static and high-rate loading of single and multi-bolt carbon-epoxy aircraft fuselage joints. Compos. Part A Appl. Sci. Manuf. 53, 97-108 (2013)

10. Zuo, Y., Cao, Z., Cao, Y., Zhang, Q., Wang, W.: Dynamic behavior of CFRP/Ti single-lap pinned joints under longitudinal electromagnetic dynamic loading. Compos. Struct. 184, 362-371 (2018)

11. Xiao, Y., Ishikawa, T.: Bearing strength and failure behavior of bolted composite joints (part I: experimental investigation). Compos. Sci. Technol. 65, 1022-1031 (2005)

12. Freedman, R.N.: A study of pull-through failures of mechanically fastened joints, MSc Thesis. Naval Postgraduate School (1977)

13. Banbury, A., Kelly, D.W.: A study of fastener pull-through failure of composite laminates. Part 1: experimental. Compos. Struct. 45, 241-254 (1999)

14. Kelly, G., Hallström, S.: Strength and failure mechanisms of composite laminates subject to localised transverse loading. Compos. Struct. 69, 301-314 (2005)

15. Catalanotti, G., Camanho, P.P., Ghys, P., Marques, A.T.: Experimental and numerical study of fastener pullthrough failure in GFRP laminates. Compos. Struct. 94, 239-245 (2011)

16. Adam, L., Bouvet, C., Castanié, B., Daidié, A., Bonhomme, E.: Discrete ply model of circular pull-through test of fasteners in laminates. Compos. Struct. 94, 3082-3091 (2012)

17. Ćwik, T., Iannucci, L., Effenberger, M.: Pull-through performance of carbon fibre epoxy composites. Compos. Struct. 94, 3037-3042 (2012)

18. Zhao, T., Palardy, G., Villegas, I.F., Rans, C., Martinez, M., Benedictus, R.: Mechanical behaviour of thermoplastic composites spot-welded and mechanically fastened joints: a preliminary comparison. Compos. Part B Eng. 112, 224-234 (2017)

19. ASTM D7332, Standard Test Method for Measuring the Fastener Pull-through Resistance of a FiberReinforced Polymer Matrix Composite, ASTM International, West Conshohocken, PA, (2007)

20. DODSSP MIL-HDBK-17-1F, Composite Materials Handbook, Polymer Matrix Composites — Guidelines for Characterization of Structural Materials, (2002)

21. Pearce, G.M.K., Johnson, A.F., Hellier, A.K., Thomson, R.S.: A study of dynamic pull-through failure of composite bolted joints using the stacked-shell finite element approach. Compos. Struct. 118, 86-93 (2014) 
22. Toso, N.R.S.: Contribution to the Modelling and Simulation of Aircraft Structures Impacting on Water, PhD Thesis, University of Stuttgart (2009)

23. Heimbs, S., Hoffmann, M., Waimer, M., Schmeer, S., Blaurock, J.: Dynamic testing and modelling of composite fuselage frames and fasteners for aircraft crash simulations. Int. J. Crashworthiness. 18, 406-422 (2013)

24. Perogamvros, N., Mitropoulos, T., Lampeas, G.: Drop tower adaptation for medium strain rate tensile testing. Exp. Mech. 56, 419-436 (2016)

25. DASSAULT Aviation. http://www.dassault-aviation.com

26. Perogamvros, N., Lampeas, G.: Experimental investigation of composite lockbolt fastened joints under inplane low velocity impact. Compos. Part A Appl. Sci. Manuf. 90, 510-521 (2016)

27. Sicuranza, G.L.: In: Circuits and Systems Tutorials (Editors Toumazou C, Battersby N and Porta S) (ed.) Theory and Approximation of Polynomial Filters, pp. 51-58. IEEE (1996)

28. Buckley, C.P., Harding, J., Hou, J.P., Ruiz, C., Trojanowski, A.: Deformation of thermosetting resins at impact rates of strain. Part I: experimental study. J. Mech. Phys. Solids. 49, 1517-1538 (2001)

29. Gerlach, R., Siviour, C.R., Petrinic, N., Wiegand, J.: Experimental characterisation and constitutive modelling of RTM-6 resin under impact loading. Polymer. 49, 2728-2737 (2008)

30. Perogamvros, N.G., Lampeas, G.N.: Experimental and numerical investigation of AS4/8552 interlaminar shear strength under impact loading conditions. J. Compos. Mater. 50, 2669-2685 (2016)

Publisher's Note Springer Nature remains neutral with regard to jurisdictional claims in published maps and institutional affiliations. 